http://www.ftsm.ukm.my/apjitm

Asia-Pacific Journal of Information Technology and Multimedia

Jurnal Teknologi Maklumat dan Multimedia Asia-Pasifik

Vol. 7 No. 1, June 2018: 83 - 89

e-ISSN: 2289-2192

\title{
THRESHOLDING AND QUANTIZATION ALGORITHMS FOR IMAGE COMPRESSION TECHNIQUES: A REVIEW
}

\author{
SAIMA ANWAR LASHARI \\ ROSZIATI IBRAHIM \\ NIK SHAHIDAH AFIFI MD TAUJUDDIN \\ NORHALINA SENAN \\ SUHAILA SARI
}

\begin{abstract}
With increasing demand on digital images, there is a need to compress the image to entertain the limited bandwidth and storage capacity. Recently, there is a growing interest among researchers focusing on compression of various types of images and data. Amongst various compression algorithms, transform-based compression is one of the promising algorithms. Despite the technological advances in transmission and storage, the demands placed on the bandwidth of communication and storage capacities by far outstrips its availability. This paper presents a review of image compression principle, compression techniques and various thresholding algorithms (pre-processing algorithms) and quantization algorithm (post-processing algorithms). This paper intends to give an overview to the relevant parties to choose the suitable image compression algorithms to suit with the need.
\end{abstract}

Keywords: Image compression, thresholding algorithm, quantization algorithm

\section{INTRODUCTION}

Image compression is a process of reducing the amount of data in an image by removing redundant data without affecting the quality of the image while keeping the resolution and Visual quality of the reconstructed image as close to the original image as possible. This minimization in size enables more image storage in an available memory space and reduces the transmission duration demanded by an image to be downloaded over the internet (Zhou et al., 2018). Image compression can basically be achieved by eliminating wherever possible various redundancies in an image. An inverse process is called decompression (decoding); applied to the compressed data to get the reconstructed image (Boujelbene et al., 2018).

Compression algorithms can be classified as either lossless or lossy compression. Lossy compression, in which the used algorithms are Fractal coding, Lossy predictive coding, vector quantization, Block Truncation coding, Transformation coding, Discrete Wavelet Transform (DWT) and subband coding. Lossless compression algorithms are Lossless Predictive Coding, Low Complexity Lossless Compression for Images (LOCO-I), Run Length Encoding, Huffman Encoding, Lempel-Ziv-Welch (LZW) encoding, area coding and variable block size segmentation. In lossless compression schemes, the reconstructed image, after compression, is numerically identical to the original image (Taujuddin et al., 2015; Jiang, 1999).

The rest of the paper is organised as follows: Section 2 introduces thresholding algorithm for image compression whilst Section 3 discusses quantization algorithm for image compression in detail. Finally, the concluding remarks are given in Section 4.

\section{THRESHOLDING ALGORITHM FOR IMAGE COMPRESSION}

Thresholding is a process of shrinking the small absolute coefficients value while retaining the 
large absolute coefficient value. It will produce a finer reconstructed signal. Since this algorithm takes the condition that the amplitude of wavelet transform coefficients signals are much larger than noises, the unconsidered noise will be removed while holding the significant signal. Threshold can also be defined as the Peak Absolute Error (PAE) accepted for image reconstruction (Baligar, Patnaik, \& Nagabhushana, 2006). Hard and soft threshold are the common operators used in conjunction with Discrete Wavelet Transform (DWT) (Zhen \& Su, 2010; Lashari Ibrahim, \& Senan. 2014). Furthermore, wavelets give superb compression ratio with the image quality maintained, which rivals Discrete Cosine Transform (DCT).

Donoho (1995) revealed that the best estimation error at its minimum is achieved through the Donoho universal threshold. Thresholding also eliminates noises and clean signals, creating some artifact. Hard threshold algorithm will preserve a good edge. However, the artifact appearance such as ringing and pseudo-Gibbs effect is irritated. The soft threshold is to lessen the gap between the remaining and discarded coefficients, giving better image recovery whereas soft threshold algorithm also produces a much better image quality over hard threshold because the end is linked, but it tends to produce an over-smoothing image (Chang, Yu \& Vetterli, 2000).

The soft threshold image appears smoother but the image is distorted, leading to a blurred image. The hard threshold eliminates all coefficients that are lower than the threshold value and maintained the values of the rest. The soft threshold ascended the coefficients continually with the centre of zero (Jianmin \& Shibo, 2009). Although there is a huge number of approaches proposed in literature, developing a threshold algorithm that reduces the image size without tolerating with the image quality is still a challenging task. Haar Wavelet, Embedded Zerotree Wavelet (EZW) and Set Partitioning in Hierarchical Tree (SPHIT) are three wavelet algorithms mostly utilised in the processing of images. Wavelet different reduction (WDR) and Adaptive Scanned Wavelet Different Reduction (ASWDR), on the other hand, are the advanced versions acknowledged in the committee. These are among the most outstanding compression algorithms which provide the smallest error per compression rate but transmitted the best perceptual quality image (Hashemi-Berenjabad, Mahloojifar \& Akhavan, 2011; Bekhtin. 2011: Lashari, Ibrahim \& Senan. 2015).

\section{RELATED WORK IN THRESHOLDING ALGORITHMS}

This section encompasses related work to wavelet threshold algorithms for image compression. Thresholding is a process of shrinking the small absolute coefficients value while retaining the large absolute coefficient value. The small absolute coefficients value is usually caused by noise while large absolute coefficients value is triggered by significant features. Generally, most of the wavelet coefficients are concentrated at near zero value with a small value. But the trend is differing at each particular subband as it associated to its resolution. Approximately $98 \%$ of coefficients in wavelet subband are smaller than $0.3 \mathrm{~W}$ where $\mathrm{W}$ denoting the largest coefficients value (Auli-Llinas, 2013). A proper elimination of near zero coefficient will not harm the significant information. Besides, by performing thresholding process, many coefficients can be set to zero which resulting in a lesser space and reduce the image size (Sztyber \& Pobocha, 2015). Threshold value estimation is very crucial in image compression. If the threshold value is set too small, it will adopt noise into data and compression ratio is not decreased. Whereas, if the threshold value is set too high, it will increase the compression ratio, but the important coefficients value will be screened out, leading to a destructive data condition (Zhen \& Su 2010). The determination of threshold values can be done by defining it manually. The widely used technique are Psychovisual threshold technique or fixed threshold technique.

Psychovisual threshold is a threshold method used to obtain the threshold value based on human observation. Usually experiments are conducted to manually detect the threshold 
value based on Just Noticeable Different (JND) point in colour space, compressibility or visual distortion of compressed image compared to original image (Ernawan et al., 2013).

Sreelekha \& Sathidevi, (2010) used the psychovisual threshold to detect the threshold on image subband. The experiment was done manually by recursively search the highest possible threshold value where the Human Visual System (HVS) cannot detect any changes or degradation of image quality. Then, each image coefficient is compared to this value, where coefficients with a higher value than the psychovisual threshold will be retained while the rest will be discarded. Although this algorithm can suggest a good threshold value below visual noticeable change, it suffer with time consuming because of manual testing. The similar approach was implemented by Abu et al. (2013), however, they implement it in DCT domain and the threshold is applied in a global condition. It then merged with the development of quantization stage. The threshold value is incremented one by one, manually, until it reaches a noticeable difference on color space (HSV). By using this threshold value, a new quantization table was developed. The resulting compressed image shows good quality, but still, it is time consuming.

Shanavaz \& Mythili (2010) gave a different view in compressing the image using fixed threshold method. They suggest an algorithm of fixing the percentage of wavelet coefficients to be zeroed. Although this algorithm offer higher compression ratio, this algorithm cause low image quality for the threshold value set more than $50 \%$ coefficients to be zeroed. Tan \& Tan, (2012) proposed another solution, where they suggested a method by fixing the reference points coding for thresholding. In this method, the threshold value is selected during compression process. Scanning was done until it meets the preferable compression ratio. This method is simple and the computational complexity is low, but compression ratio has to be compensated with degradation of image quality and error occurred during scanning will propagate threshold value. Some similar researches also have been done by (Palzer \& Timo, 2016). To sum up, Table 1 present the summary of thresholding techniques as discussed in this section.

TABLE 1. Summary of Thresholding Techniques

\begin{tabular}{|c|c|c|c|c|c|c|}
\hline \multirow{2}{*}{ Technique } & \multirow{2}{*}{ Author } & \multicolumn{2}{|c|}{ Types } & \multirow{2}{*}{$\begin{array}{l}\text { Data } \\
\text { sample }\end{array}$} & \multirow{2}{*}{ Advantage } & \multirow{2}{*}{ Disadvantage } \\
\hline & & Global & Local & & & \\
\hline & $\begin{array}{l}\text { Sreelekha \& } \\
\text { Sathidevi, } \\
2010\end{array}$ & & $\sqrt{ }$ & $\begin{array}{l}\text { RGB } \\
\text { image }\end{array}$ & $\begin{array}{l}\text { Threshold } \\
\text { value is below } \\
\text { noticeable } \\
\text { change }\end{array}$ & Time consuming \\
\hline
\end{tabular}

Psychovisual

\begin{tabular}{|c|c|c|c|c|}
\hline $\begin{array}{l}\text { Abu et al., } \\
2013\end{array}$ & $\sqrt{ }$ & $\begin{array}{l}\text { Grey } \\
\text { scale } \\
\text { image }\end{array}$ & $\begin{array}{l}\text { Good image } \\
\text { quality }\end{array}$ & $\begin{array}{l}\text { Manual increment on } \\
\text { threshold value }\end{array}$ \\
\hline $\begin{array}{l}\text { Baligar, } \\
2006\end{array}$ & $\sqrt{ }$ & $\begin{array}{l}\text { Grey } \\
\text { scale } \\
\text { image }\end{array}$ & $\begin{array}{l}\text { Low } \\
\text { complexity }\end{array}$ & $\begin{array}{l}\text { Fit on certain image } \\
\text { category only }\end{array}$ \\
\hline $\begin{array}{l}\text { Shanavaz \& } \\
\text { Mythili, } \\
2010\end{array}$ & $\sqrt{ }$ & $\begin{array}{l}\text { Finger } \\
\text { print } \\
\text { image }\end{array}$ & $\begin{array}{l}\text { High } \\
\text { Compression } \\
\text { Ratio (CR) }\end{array}$ & $\begin{array}{l}\text { Quality degrade if } \\
\text { threshold } \\
\begin{array}{l}\text { zeroed } \\
\text { coefficients }\end{array}\end{array}$ \\
\hline
\end{tabular}

Fixed 


\begin{tabular}{|c|c|c|c|c|c|}
\hline $\begin{array}{l}\text { Tan \& Tan, } \\
2012\end{array}$ & $\sqrt{ }$ & & $\begin{array}{l}\text { Grey } \\
\text { scale } \\
\text { image }\end{array}$ & $\begin{array}{l}\text { Various } \\
\text { threshold } \\
\text { value }\end{array}$ & $\begin{array}{lr}\text { Error } & \text { during } \\
\text { scanning } & \text { will } \\
\text { propagate } & \text { threshold } \\
\text { value } & \end{array}$ \\
\hline $\begin{array}{l}\text { Abirami } \\
\text { al., } 2013\end{array} \quad$ et & & $\sqrt{ }$ & $\begin{array}{l}\text { Grey } \\
\text { scale } \\
\text { image }\end{array}$ & $\begin{array}{l}\text { Flexibility in } \\
\text { choosing } \\
\text { threshold } \\
\text { value }\end{array}$ & $\begin{array}{l}\text { No significant } \mathrm{CR} \\
\text { change after } 4^{\text {th }} \text { level } \\
\text { decomposition }\end{array}$ \\
\hline $\begin{array}{l}\text { Palzer \& } \\
\text { Timo, } 2016\end{array}$ & $\sqrt{ }$ & & $\begin{array}{l}\text { Grey } \\
\text { scale } \\
\text { image }\end{array}$ & $\begin{array}{l}\text { Multiple } \\
\text { threshold } \\
\text { value }\end{array}$ & $\begin{array}{l}\text { High CR will cause } \\
\text { low PSNR }\end{array}$ \\
\hline
\end{tabular}

While, fixed threshold is a very simple threshold method where the threshold value is predefined at the pre-processing stage. Formerly, Baligar suggested the use only one fixed threshold value to be apply globally to an image (Baligar, Patnaik \& Nagabhushana, 2006) so it just can fit on certain image category only. While, Abirami et al., (2013) extend this work by suggesting the use of multiple threshold value then select the one that provide the highest Peak Signal-to-Noise Ratio (PSNR) value. These algorithms provide a manual suggestion to select the threshold value. Although it is fast, on the other hand it will limit the performance of compression because it creates a frontier where the coefficients cannot be reduced higher than the permanent limit.

\section{QUANTIZATION ALGORITHM FOR IMAGE COMPRESSION}

Quantization is a significant part in compression, charting the integer value of the quantized data to the tone value which is represented by some index value. High speed execution time is created but conversely, high quantization error is also produced because of the rounding process (Abu, Ernawan \& Suryana, 2013). The precision of floating point value of wavelet coefficient is lessened by the quantization process. These approximated values are quantized, thus creating a lossy compressed image.

Defining the interval size for quantization process is very essential as it will provide compact signal representations with as little information loss as possible. Thus, the motivation of recent research is to define interval that gives good discrimination between significant and non-significant coefficient. By adaptively change the quantization interval size, original data used more than information. But the challenge is in formulating a virtuous quantization algorithm that can achieve high compression ratio without loss its reliability. Compression algorithm will end up with encoding algorithm which usually uses statistical technique to minimize redundancy. At this stage, the symbol stream will be replaced by sequence of binary code word, which is the smallest possible number of bit per symbol.

Due to high performance in achieving minimum code length, Huffman (Abu, Ernawan, \& Suryana, 2013) is the most commonly used technique. Another two techniques that usually used for encoding are RLE and Arithmetic Coding Savić, Perić \& Simić (2015) in their research proposed dual mode quantization by using low and medium number of quantization levels and fixing the code word length by using pixel value prediction in the pre-processing stage. Prediction is done on blocks with $\mathrm{m} \times \mathrm{m}$ sizes. Linear prediction is performed by calculating the variance between original and predicted block, followed by fixed uniform or piecewise uniform quantization and differential encoding. 
The Uniform Scalar Zero Zone Quantization (USZZQ) performs uniform quantization at valued coefficient range with same interval size, while double the interval size at zero coefficient range. Bartrina-rapesta \& Auli-llinas (2015) proposed an extended version of USDQ named Two-Step Scalar Deadzone Quantization (2SDQ). The study used two different size of interval at valued coefficient range based on its magnitude. Coefficients with larger magnitude will have larger interval size compared to smaller coefficient magnitude. Besides, these algorithms depend on a small set of Wavelet coefficient magnitudes called cell.

Mahapatra \& Jena (2013) presented a generalised fuzzy c-means clustering approach GIFP-FCM. This clustering approach, when applied to WQ based image compression, suitably demonstrates that the transition from fuzzy to crisp mode is more efficient compared to the known approaches. It is also independent of the choice of the initial codebook vector. The technique is fast, easy to implement and has rapid convergence. Bhattacharyya et al. 2007, proposed Hybrid DCT-VQ, the concept of energy compaction property of DCT to decrease the computational cost of codebook and to increase the VQ process. The simulation showed better results in terms of execution time and compression ratio as compared to that of conventional VQ process. Table 2 depicts the summary of the algorithms mentioned in this section.

TABLE 2. Quantization Algorithms Proposed By Recent Researchers

\begin{tabular}{|c|c|c|c|}
\hline Authors & Technique & Advantage & Disadvantage \\
\hline Savic et al., 2015 & Dual Mode Quantization & $\begin{array}{c}\text { High Peak Signal to } \\
\text { Quantization Noise Ratio } \\
\text { (PSQNR) }\end{array}$ & $\begin{array}{l}\text { High bit allocation needed } \\
\text { for less correlated image }\end{array}$ \\
\hline $\begin{array}{l}\text { Manikandan \& } \\
\text { Dandapat, } 2007\end{array}$ & $\begin{array}{l}\text { Uniform Scalar Zero } \\
\text { Zone Quantizer }\end{array}$ & $\begin{array}{c}\text { Provide energy packing } \\
\text { efficiency }\end{array}$ & Suitable only on ECG signal \\
\hline $\begin{array}{l}\text { Bartrina-rapesta \& Auli- } \\
\text { Ilinas, } 2015\end{array}$ & $\begin{array}{c}\text { Two-step Scalar } \\
\text { Deadzone Quantizer }\end{array}$ & Produce high quality image & $\begin{array}{c}\text { Auxiliary information } \\
\text { transferred at low bit rate } \\
\text { cause lower PSNR value at } \\
\text { this rate }\end{array}$ \\
\hline Mahapatra \&Jena,2013 & - & High speed performance & Difficult to implement \\
\hline $\begin{array}{c}\text { Bhattacharyya, } \\
2007\end{array}$ & Hybrid DCT-VQ & $\begin{array}{l}\text { Better execution time and } \\
\text { compression ratio }\end{array}$ & - \\
\hline
\end{tabular}

Although these algorithms produce good image quality, it suffers with timeconsumption because of various steps introduced in the algorithm. Besides, in the prediction part, manual examination is used.

\section{CONCLUSION}

Nowadays, wavelet-based compression with its multi-resolution representation becomes a leading technology. In addition, it delivers impressive reconstruction image and more ideas can be developed for image compression. There are many generic wavelets being introduced currently. Sophisticated wavelets produce a smoother and more satisfactory compressed image. It makes no assumption concerning the periodicity of the data. Thus, wavelets are suitable for demonstrating sharp changes or even discontinuities. In this paper, several compression techniques for lossless and lossy compression techniques are presented. Current compression algorithms present an excellent performance, and many of them support the use of wavelet transformation. The previous researches show that thresholding ad quantization gives 
significant impact on the quality of the compressed image. Higher threshold value will give higher compression ratio but the image quality will tend to decrease, and vice-versa. Besides, appropriate decision on interval size in quantization is needed in reducing the quantization error because it also can affect the final reconstructed image. Despite the fact that great efforts are made to seek image compression at modest complexity and efficient performance, it still faces many challenges in achieving various degrees of scalability at different target bit rate based on users' individual requirements.

\section{REFERENCES}

Abirami, J., Narashiman, K., SivaSankari, S. and Ramya, S., 2013. April. Performance anaysis of image compression using wavelet thresholding. In Information \& Communication Technologies (ICT), 2013 IEEE Conference on (pp. 194-198). IEEE.

Abu, N.A., Ernawan, F. and Suryana, N., 2013, March. A generic psychovisual error threshold for the quantization table generation on JPEG image compression. In Signal Processing and its Applications (CSPA), 2013 IEEE 9th International Colloquium on(pp. 39-43). IEEE.

Auli-Llinas, F., 2013. 2-step scalar deadzone quantization for bitplane image coding. IEEE Transactions on Image Processing, 22(12), pp.4678-4688.

Baligar, V.P., Patnaik, L.M. and Nagabhushana, G.R., 2006. Low complexity, and high fidelity image compression using fixed threshold method. Information Sciences, 176(6), pp.664-675.

Bartrina-Rapesta, J. and Auli-Llinas, F., 2015. Cell-based two-step scalar deadzone quantization for high bit-depth hyperspectral image coding. IEEE Geoscience and Remote Sensing Letters, 12(9), pp.1893-1897.

Bekhtin, Y.S., 2011, September. Adaptive wavelet codec for noisy image compression. In Design \& Test Symposium (EWDTS), 2011 9th East-West (pp. 184-188). IEEE.

Bhattacharya, C., \& Mahapatra, P. R. 2007. A discrete wavelet transform approach to multiresolution complex SAR image generation. IEEE Geoscience and Remote Sensing Letters, 4(3), 416-420.

Boujelbene, R., Jemaa, Y. B., \& Zribi, M. 2018, A comparative study of recent improvements in wavelet-based image coding schemes. Multimedia Tools and Applications, 1-35.

Chang, S.G., Yu, B. and Vetterli, M., 2000. Adaptive wavelet thresholding for image denoising and compression. IEEE transactions on image processing, 9(9), pp.1532-1546.

Chen, R.C., Pai, P.Y., Chan, Y.K. and Chang, C.C., 2009. Lossless image compression based on multiple-tables arithmetic coding. Mathematical problems in Engineering, 2009.

Chrysafis, C. and Ortega, A., 2000. Line-based, reduced memory, wavelet image compression. IEEE Transactions on Image processing, 9(3), pp.378-389.

Donoho, D.L., 1995. De-noising by soft-thresholding. IEEE transactions on information theory, 41(3), pp.613-627.

Ernawan, F., Abu, N.A. and Suryana, N., 2013, March. TMT quantization table generation based on psychovisual threshold for image compression. In Information and Communication Technology (ICoICT), 2013 International Conference of (pp. 202-207). IEEE.

Hashemi-Berenjabad, S., Mahloojifar, A. and Akhavan, A., 2011, December. Threshold based lossy compression of medical ultrasound images using contourlet transform. In Biomedical Engineering (ICBME), 2011 18th Iranian Conference of (pp. 191-194). IEEE.

Iwahashi, M., Yoshida, T. and Kiya, H., 2014, December. Range reduction of HDR images for backward compatibility with LDR image processing. In Asia-Pacific Signal and Information Processing Association, 2014 Annual Summit and Conference (APSIPA) (pp. 1-4). IEEE.

Jiang, J., 1999. Image compression with neural networks-a survey. Signal Processing: Image Communication, 14(9), pp.737-760.

Jianmin, L. and Shibo, X., 2009, November. Analysis and application of modified methods of wavelet threshold functions. In Computational Intelligence and Industrial Applications, 2009. PACIIA 2009. Asia-Pacific Conference on (Vol. 1, pp. 150-153). IEEE.

Lashari, S.A., Ibrahim, R. and Senan, N., 2014, December. De-noising analysis of mammogram images in the wavelet domain using hard and soft thresholding. In Information and Communication Technologies (WICT), 2014 Fourth World Congress on (pp. 353-357). IEEE. 
Lashari, S.A., Ibrahim, R. and Senan, N., 2015. Wavelet Threshold De-Noising for Mammogram Images. International Journal of Software Engineering and Its Applications, 9(6), pp.215-226.

Mahapatra, D.K. and Jena, U.R., 2013, April. Partitional k-means clustering based hybrid DCT-Vector Quantization for image compression. In Information \& Communication Technologies (ICT), 2013 IEEE Conference on (pp. 1175-1179). IEEE.

Palzer, L. and Timo, R., 2016, July. Fixed-length compression for letter-based fidelity measures in the finite blocklength regime. In Information Theory (ISIT), 2016 IEEE International Symposium on(pp. 2424-2428). IEEE.

Savić, M.S., Perić, Z.H. and Simić, N., 2015. Coding algorithm for grayscale images based on Linear Prediction and dual mode quantization. Expert Systems with Applications, 42(21), pp.72857291.

Shanavaz, K.T. and Mythili, P., 2010, October. An improved technique for evolving wavelet coefficients for fingerprint image compression. In Communication Control and Computing Technologies (ICCCCT), 2010 IEEE International Conference on(pp. 665-669). IEEE.

Sreelekha, G. and Sathidevi, P.S., 2010. An HVS based adaptive quantization scheme for the compression of color images. Digital Signal Processing, 20(4), pp.1129-1149.

Srikanth, S. and Meher, S., 2013, April. Compression efficiency for combining different embedded image compression techniques with Huffman encoding. In Communications and Signal Processing (ICCSP), 2013 International Conference on (pp. 816-820). IEEE.

Sztyber, A. and Pobocha, M., 2015, September. Comparison of wavelet thresholding methods for industrial data compression. In Intelligent Data Acquisition and Advanced Computing Systems: Technology and Applications (IDAACS), 2015 IEEE 8th International Conference on (Vol. 1, pp. 192-197). IEEE.

Tan, Y.F. and Tan, W.N., 2012, July. Image compression technique utilizing reference points coding with threshold values. In Audio, Language and Image Processing (ICALIP), 2012 International Conference on (pp. 74-77). IEEE.

Taujuddin, N.S.A.M., Ibrahim, R. and Sari, S., 2015, April. Image compression using a new adaptive standard deviation thresholding estimation at the wavelet details subbands. In Computing Technology and Information Management (ICCTIM), 2015 Second International Conference on (pp. 109-114). IEEE.

Tay, P.C., Acton, S.T. and Hossack, J.A., 2011. A wavelet thresholding method to reduce ultrasound artifacts. Computerized Medical Imaging and Graphics, 35(1), pp.42-50.

Yadav, R.J., Gangwar, S.P. and Singh, H.V., 2012. Study and analysis of wavelet based image compression techniques. International Journal of Engineering, Science and Technology, 4(1), pp.1-7.

Zhen, C. and Su, Y., 2010, June. Research on wavelet image threshold de-noising. In Future Power and Energy Engineering (ICFPEE), 2010 International Conference on (pp. 3-6). IEEE.

Zhou, Q., Yao, H., Cao, F., \& Hu, Y. C. 2018. Efficient image compression based on side match vector quantization and digital inpainting. Journal of Real-Time Image Processing, 1-12.

Saima Anwar Lashari

Department of Computer Sciences

University of Lahore, Sargodha Campus

Sargodha, Pakistan.

saima.anwar@cs.uol.edu.pk

Rosziati Ibrahim,

Nik Shahidah Afifi Md Taujuddin

Norhalina Senan

Suhaila Sari

Fakulti Sains Komputer Dan Teknologi Maklumat

Universiti Tun Hussein Onn Malaysia

Received: 17 December 2017

Accepted: 3 February 2018

Published: 26 June 2018 